Research Article

\title{
Adaptive Fuzzy Type-II Controller for Wheeled Mobile Robot with Disturbances and Wheelslips
}

\author{
Viet Quoc Ha, ${ }^{1}$ Sen Huong-Thi Pham, ${ }^{2}$ and Nga Thi-Thuy Vu $\mathbb{D}^{1}$ \\ ${ }^{1}$ School of Electrical Engineering, Hanoi University of Science and Technology, Hanoi, Vietnam \\ ${ }^{2}$ Faculty of Control and Automation, Electric Power University, Hanoi, Vietnam \\ Correspondence should be addressed to Nga Thi-ThuyVu; nga.vuthithuy@hust.edu.vn
}

Received 9 June 2021; Revised 26 July 2021; Accepted 30 August 2021; Published 15 September 2021

Academic Editor: Arturo Buscarino

Copyright ( 2021 Viet Quoc Ha et al. This is an open access article distributed under the Creative Commons Attribution License, which permits unrestricted use, distribution, and reproduction in any medium, provided the original work is properly cited.

This paper proposed adaptive fuzzy type-II controllers for the wheeled mobile robot (WMR) systems under conditions of wheel slips and disturbances. The system includes two control loops: outer loop for position tracking and the inner loop for velocity tracking. In each loop, the controller has two parts: the feedback which keeps the system stable and the adaptive type-II fuzzy part which is used to compensate the unknown components that act on the system. The stability of each loop as well as the overall system is proven mathematically based on the Lyapunov theory. Finally, the simulation is setup to verify the effectiveness of the presented algorithm. The simulation results show that, in comparison with the corresponding fuzzy type-I controller, the performance of the adaptive fuzzy type-II controller is better, i.e., the position error is smaller and the velocity is almost smooth under the conditions that the reference trajectory is changed, and the system is affected by wheel slips and external disturbances.

\section{Introduction}

Wheeled mobile robots (WMRs) are widely used in industry and service robotics. Mobile robots are self-moving vehicles and versatile with many indoor and outdoor applications [1]. The control problems in the WRMs are quite rich such as path planning, trajectory tracking, and obstacle avoidance. Each problem has different importance in specific application. Among these, trajectory tracking control has the role to keep the WMR following the desired trajectory. This control problem is not easy because WMRs are underactuated systems. Moreover, operation of WMR is greatly affected by working conditions such as system uncertainties, wheel slips, and external disturbances. Therefore, tracking control design for WRM still has the attraction to many researchers.

Many nonlinear control methods have been used to solve the tracking control problems of nonholonomic mobile robots such as robust adaptive [2], sliding mode control $[3,4]$, backstepping control $[5,6]$, adaptive fuzzy logic control [7], and adaptive neural-network control [8,9]. However, all of these are proposed with the assumption of "pure rolling without slip." As mentioned above, these assumptions are not exactly since there are many factors such as unknown external disturbances and wheel slipping due to the slippery roads and the wheel. In order to reduce the effect of the wheel slips, an adaptive robust control is used for trajectory tracking of wheeled mobile robots in the presence of unknown skidding and slipping [10]. Although this controller solved external disturbances, the error tracking is still pretty big. In [11], the adaptive fuzzy output feedback controller is proposed to solve the trajectory problem for WMR with the uncertainties and effects of external disturbances. In this research, the tracking position errors converge asymptotically to a small neighborhood near the origin with a faster response than achieved by other existing controllers, and all of the signals are bounded. A controller based on the robust dynamic surface control method is proposed in [12] to eliminate the problems of "explosion of complexity." The controller can become much simpler than backstepping controller, but the illustrated results are so poor. An adaptive neural network based on reinforcement learning is presented in [13] for WMR with considering skidding and slipping. In this work, the error tracking is 
almost zero, but the structure with four neural networks in the scheme can be the burden for calculation system.

Recently, the advanced and intelligent control methods are considered as flexible tools to deal with the uncertain systems. In [14], the authors propose a strategy that can ensure optimal working under the imperfect dynamic conditions based on the excitation of the hidden dynamics. Nevertheless, some strict assumptions need to be satisfied to complete the control strategy. In [15], the disturbance problems for nonlinear systems are solved by using neural networks. This controller guarantees that all the signals in the closed-loop system are semiglobally uniformly ultimate bounded. However, due to the effect of the iterative derivation of virtual control laws, the "complexity explosion" problem appears which increases the computational complexity. Another approach to deal with the model parameter uncertainty problem is shown in [16]. In this research, a model is developed for corrosion prediction using a neurofuzzy expert system. The results show that the neurofuzzy expert systems have better accuracy with fewer number of input parameters than the neural networks prediction model. In [17], a controller based on the fuzzy logic system to approximate unknown parameters in nonlinear systems is proposed. In this work, the tracking error can converge to a small neighborhood of zero with a fixed time, and all the signals of the system are bounded. However, the external disturbances are not considered in this research. In $[18,19]$, the interval fuzzy type-II controller is introduced for nonlinear uncertain systems. It is proved that the fuzzy type-II controller handles uncertainties and external disturbances better than the fuzzy type-I controller.

In this paper, an adaptive interval type-2 fuzzy logic controller is proposed for two-loop control of wheeled mobile robots with external disturbances. The proposed controller is expected to allow the error tracking of WMRs to converge to zero under the acting of unknown wheel slips, unknown bounded external disturbances, and model uncertainties.

The main contributions of the proposed algorithm can be stated as follows:

(i) Design the adaptive interval fuzzy type-II controller for both the inner and outer loop of WMR with uncertainties and external disturbances.

(ii) Under the conditions that the reference trajectory is complex changed, the proposed controller can still deal with the tracking problem, and the result showed that the error between the reference trajectory and the real trajectory is quite small.

(iii) Using the adaptive fuzzy type-II controller, the difference between the real velocity and the reference velocity is almost zero. Moreover, in this research, the comparison results show that the real velocity of the proposed controller is smoother than the real velocity of the fuzzy type-I controller. (iv) The stability of the closed-loop system with the proposed controller is proven mathematically based on the Lyapunov theory. This proof for the cascade system is much harder than the single loop.

\section{System Model and Adaptive Controller Design}

2.1. System Modeling. Let consider a nonholonomic WMR with skidding and slipping as shown in Figure 1. Located at point $G\left(x_{G}, y_{G}\right)$ is the center of mass (COM) of the wheel mobile robot, and point $M\left(x_{M}, y_{M}\right)$ indicates the midpoint of the wheel shaft. The distance between $G$ and $M$ is $a . r$ is the radius of each driving wheel, and the length of wheel mobile robot shaft is $2 b$. $\theta$ is the orientation of the wheel mobile robot with respect to the initial frame.

$F_{1}$ and $F_{2}$ illustrate the total longitudinal friction force at the right and left driving wheels, respectively. $F_{3}$ is the total lateral force that acts on the mobile robot, whereas $F_{4}$ and $₫$ are the external disturbances and moment at point $G$ accordingly.

According to [20], we can consider the kinematics model of the wheeled mobile robot under slipping and skidding condition as follows:

$$
\left\{\begin{array}{l}
\dot{x}_{M}=\vartheta \cos \theta-\dot{\eta} \sin \theta, \\
\dot{y}_{M}=\vartheta \sin \theta+\dot{\eta} \cos \theta, \\
\dot{\theta}=\omega,
\end{array}\right.
$$

where $\vartheta$ is the forward velocity and $\omega$ is the angular velocity of the wheeled mobile robot at points $M, \vartheta$, and $\omega$ and are calculated as follows:

$$
\begin{aligned}
& \vartheta=\frac{r\left(\dot{\phi}_{R}+\dot{\phi}_{L}\right)}{2}+\frac{\dot{\gamma}_{R}+\dot{\gamma}_{L}}{2}, \\
& \omega=\frac{r\left(\dot{\phi}_{R}-\dot{\phi}_{L}\right)}{2 b}+\frac{\dot{\gamma}_{R}-\dot{\gamma}_{L}}{2 b},
\end{aligned}
$$

with $\gamma_{R}$ and $\gamma_{L}$ are the coordinates of the longitudinal slip of the right and left driving wheels, respectively, and $\eta$ is the coordinate of the lateral slip along the wheel shaft. $\dot{\varphi}_{R}$ and $\dot{\varphi}_{L}$ are the angular velocities of the right and the left wheels, respectively.

We consider a two-wheeled mobile robot with coordinates illustrated in Figure 1 described by the following dynamic models [20]:

$$
M \dot{v}+B(v) v+E v+Q \ddot{\gamma}+C \dot{\eta}+G \ddot{\eta}+\tau_{d}=\tau,
$$

where $\quad v=\left[\begin{array}{ll}\dot{\phi}_{R} & \dot{\phi}_{L}\end{array}\right]^{T}, \quad \gamma=\left[\begin{array}{ll}\gamma_{R} & \gamma_{L}\end{array}\right]^{T}, \quad$ and $\quad M=$ $\left[\begin{array}{ll}m_{11} & m_{12} \\ m_{21} & m_{22}\end{array}\right]$. 


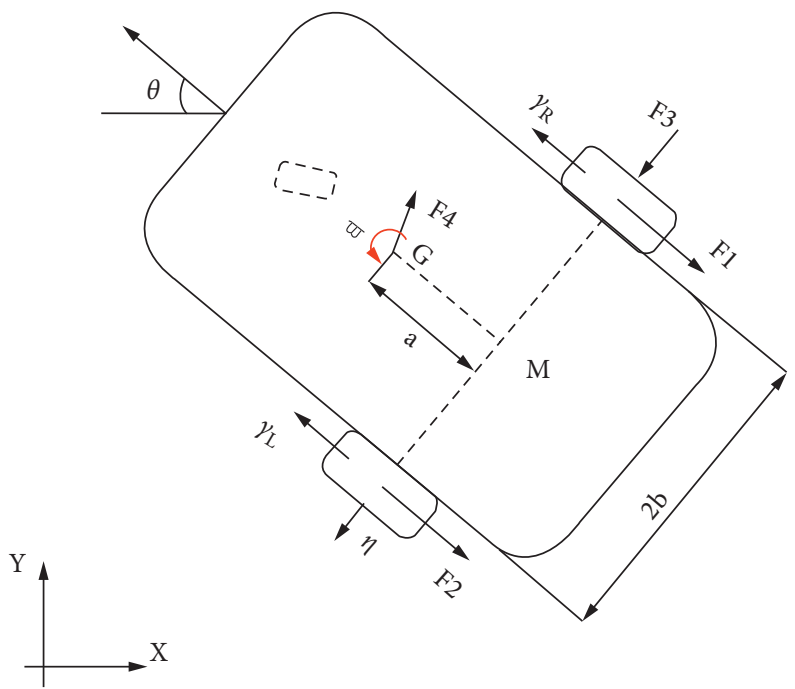

Figure 1: Model of the wheeled mobile robot.

$$
\begin{aligned}
& m_{12}=m_{21}=m_{G}\left(\frac{r^{2}}{4}-\frac{a^{2} r^{2}}{4 b^{2}}\right)-\frac{r^{2}}{4 b^{2}}\left(I_{G}+2 I_{D}\right), \\
& m_{11}=m_{22}=m_{G}\left(\frac{r^{2}}{4}+\frac{a^{2} r^{2}}{4 b^{2}}\right)+\frac{r^{2}}{4 b^{2}}\left(I_{G}+2 I_{D}\right)+m_{W} r^{2}+I_{w}, \\
& Q=\left[\begin{array}{ll}
Q_{1} & Q_{2} \\
Q_{2} & Q_{1}
\end{array}\right] \\
& Q_{1,2}=m_{G} \frac{r}{4}\left(1 \pm \frac{a^{2}}{b^{2}}\right) \pm \frac{r}{4 b}\left(I_{G}+2 I_{D}\right), \\
& B(v)=m_{G} \frac{a r^{3}}{4 b^{2}}\left(\dot{\phi}_{R}-\dot{\phi}_{L}\right)\left[\begin{array}{cc}
0 & 1 \\
-1 & 0
\end{array}\right] \\
& E=\left(m_{G} \frac{a r^{2}}{2 b}\right)\left(\frac{\dot{\gamma}_{R}-\dot{\gamma}_{L}}{2 b}\right)\left[\begin{array}{cc}
0 & 1 \\
-1 & 0
\end{array}\right], \\
& G=m_{G} \frac{a r}{2 b}\left[\begin{array}{c}
1 \\
-1
\end{array}\right] \\
& C=m_{G} \frac{r}{2}\left[\begin{array}{l}
1 \\
1
\end{array}\right] \omega, \\
& \tau=\left[\tau_{R}, \tau_{L}\right]^{T} .
\end{aligned}
$$

2.2. Adaptive Tracking Controller Design for WMR. The control objective of this work is to design the controller for the WMR subjected to unknown wheel slips and model uncertainties to ensure that the desired reference trajectory is tracked. To solve this problem, the adaptive control loop is proposed with the block diagram shown in Figure 2:
2.2.1. Kinematic Controller Design. In the $x \mathrm{O} y$ coordinate, the position error between the point $M\left(x_{M}, y_{M}\right)$ and the target point $C\left(x_{C}, y_{C}\right)$ is calculated as follows:

$$
e_{p}=\left[\begin{array}{c}
e_{p 1} \\
e_{p 2}
\end{array}\right]=\left[\begin{array}{cc}
\cos \theta & \sin \theta \\
-\sin \theta & \cos \theta
\end{array}\right]\left[\begin{array}{l}
x_{C}-x_{M} \\
y_{C}-y_{M}
\end{array}\right] \text {. }
$$




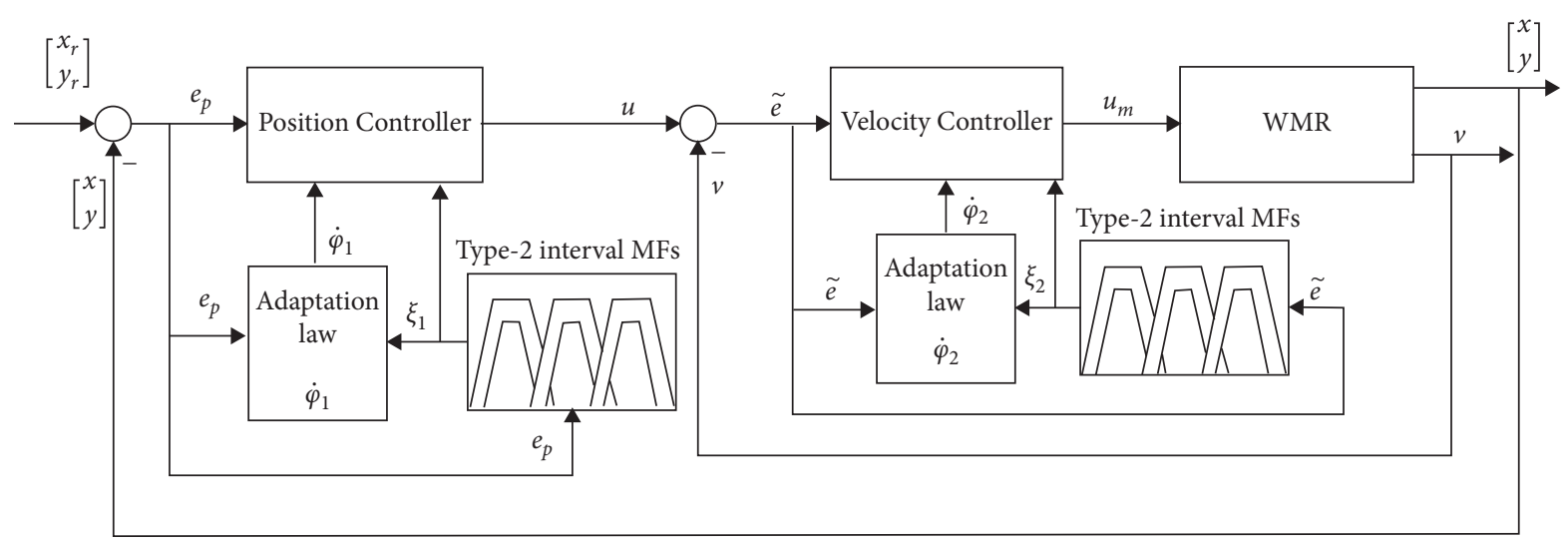

Figure 2: The control scheme for the wheeled mobile robot.

The derivative of (5) along with time under the condition of wheel slips and external disturbances is obtained as [20]

$$
\dot{e}_{p}=\left[\begin{array}{c}
\dot{e}_{p 1} \\
\dot{e}_{p 2}
\end{array}\right]=\left[\begin{array}{cc}
\cos \theta & \sin \theta \\
-\sin \theta & \cos \theta
\end{array}\right]\left[\begin{array}{c}
\dot{x}_{C} \\
\dot{y}_{C}
\end{array}\right]+h u+\mathrm{d}_{p},
$$

where $h=\left[\begin{array}{cc}\left(e_{p 2} / b-1\right) r / 2 & -\left(e_{p 2} / b+1\right) r / 2 \\ -e_{p 1} r / 2 b & e_{p 1} r / 2 b\end{array}\right]$ and $\mathrm{d}_{p}=$ $\left[\begin{array}{c}\left(\left(\left(\dot{\gamma}_{R}-\dot{\gamma}_{L}\right) / 2 b\right) e_{p 2}\right)-\left(\left(\dot{\gamma}_{R}+\dot{\gamma}_{L}\right) / 2\right) \\ -\left(\left(\left(\dot{\gamma}_{R}-\dot{\gamma}_{L}\right) / 2 b\right) e_{p 1}\right)-\dot{\eta}\end{array}\right]$

The control $u$ is separated into two components $u_{1}$ and $u_{2}$, in which $u_{1}$ is the feed-forward part used to compensate for the nonlinear component in model (6) and $u_{2}$ is the feedback controller.

$$
u=u_{1}+u_{2}
$$

where

$$
u_{1}=-h^{-1}\left[\begin{array}{cc}
\cos \theta & \sin \theta \\
-\sin \theta & \cos \theta
\end{array}\right]\left[\begin{array}{c}
\dot{x}_{C} \\
\dot{y}_{C}
\end{array}\right] .
$$

Substituting (7) and (8) into (6), we get

$$
\dot{e}_{p}=h u_{2}+\mathrm{d}_{p} \text {. }
$$

First, considering (9) without disturbances $(d=0)$, we propose the controller:

$$
u_{2}^{*}=-h^{-1} K_{1} e_{p},
$$

with $K_{1}$ is the positive scalar.

Substituting (10) into (9), we can achieve the control goal that is $\lim _{t \rightarrow \infty} e_{p}(t)=0$.

It is noted that when the WMR tracks the desired trajectory, the errors $e_{p 1}$ and $e_{p 2}$ go to zero. This leads to $\operatorname{det}(h)=-e_{p 1} r^{2} / 2 b \longrightarrow 0$ and $h$ is not invertible. To avoid this problem, controllers (8) and (10) are modified as follows:

$$
\begin{aligned}
& u_{1}= \begin{cases}-h^{-1}\left[\begin{array}{cc}
\cos \theta & \sin \theta \\
-\sin \theta & \cos \theta
\end{array}\right]\left[\begin{array}{l}
\dot{x}_{C} \\
\dot{y}_{C}
\end{array}\right], & \text { if }\left|e_{p 1}\right| \geq \lambda, \\
\frac{1}{\lambda}\left[\begin{array}{cc}
\cos \theta & \sin \theta \\
-\sin \theta & \cos \theta
\end{array}\right]\left[\begin{array}{l}
\dot{x}_{C} \\
\dot{y}_{C}
\end{array}\right], & \text { if }\left|e_{p 1}\right|<\lambda,\end{cases} \\
& u_{2}^{*}= \begin{cases}-h^{-1} K_{1} e_{p}, & \text { if }\left|e_{p 1}\right| \geq \lambda, \\
\frac{1}{\lambda} K_{1} e_{p}, & \text { if }\left|e_{p 1}\right|<\lambda .\end{cases}
\end{aligned}
$$

Here, $\lambda$ is a small enough scalar.

In fact, the disturbances are unknown $\left(\mathrm{d}_{p} \neq 0\right)$, and then we cannot apply controller (10) directly. To solve this problem, we will design the adaptive interval fuzzy type-II logic controller to approximate control law $u_{2}^{*}$ as follows:

$$
u_{2}=u\left(e_{p} \mid \varphi_{1}\right)-h^{-1}\left(\Lambda_{1} e_{p}+\Lambda_{2} \int_{0}^{t} e_{p} \mathrm{~d} t\right)
$$

where $\Lambda_{1}$ and $\Lambda_{2}$ are positive scalars and $u\left(e_{p} \mid \varphi_{1}\right)$ $=\xi_{1}^{T}\left(e_{p}\right) \varphi_{1}$ is the output of the interval fuzzy type-II logic controller in which $\xi_{1}\left(e_{p}\right)=\operatorname{diag}\left(\left[\begin{array}{lll}\xi_{11}\left(e_{p}\right) & \xi_{12} & \left(e_{p}\right), \ldots\end{array}\right.\right.$ $\left.\left.\xi_{1 M}\left(e_{p}\right)\right]\right), \xi_{1 j}=f_{1 j} / \sum_{j=1}^{M} f_{1 j}$, where $f_{1 j}$ is the firing interval of the $j$ th-rule, $M$ is the number of rules, and $\varphi_{1}$ is 
the designed parameter which is calculated by the adaptive law:

$$
\dot{\varphi}_{1}=-\left\{\xi_{1}\left(e_{p}\right) h^{T} B_{1} e_{p}\right\} .
$$

Substituting (12) into (9), it leads to the following results:

$$
\begin{aligned}
\dot{e}_{p} & =-\Lambda_{1} e_{p}-\Lambda_{2} \int_{0}^{t} e_{p} \mathrm{~d} t+h\left(u\left(e_{p} \mid \varphi_{1}\right)-u_{2}^{*}\right)+h u_{2}^{*}+\mathrm{d}_{p} \\
& =-A e_{p}-\Lambda_{2} \int_{0}^{t} e_{p} \mathrm{~d} t+B_{1} h\left(u\left(e_{p} \mid \varphi_{1}\right)-u_{2}^{*}\right)+B_{1} \Delta_{p},
\end{aligned}
$$

where $A=\Lambda_{1}+K_{1}, B_{1}=\left[\begin{array}{ll}1 & 0 \\ 0 & 1\end{array}\right]$ and $\Delta_{p}=\left[\begin{array}{ll}\mathrm{d}_{p 1} & \mathrm{~d}_{p 2}\end{array}\right]^{T}$.

Defining the estimated error:

$$
\widetilde{w}=h\left(u\left(e_{p} \mid \varphi_{1}^{*}\right)-u_{2}^{*}\right) .
$$

Substituting (15) into (14), the following is obtained:

$$
\begin{aligned}
\dot{e}_{p} & =-A e_{p}-\Lambda_{2} \int_{0}^{t} e_{p} \mathrm{~d} t+B_{1} h\left(u\left(e_{p} \mid \varphi_{1}\right)-u\left(e_{p} \mid \varphi_{1}^{*}\right)\right)+B_{1}\left(\Delta_{p}+\widetilde{w}\right) \\
& =-A e_{p}-\Lambda_{2} \int_{0}^{t} e_{p} \mathrm{~d} t+B_{1} h \xi_{1}^{T} \widetilde{\varphi}_{1}+B_{1} \sigma,
\end{aligned}
$$

where $\widetilde{\varphi}_{1}=\varphi_{1}-\varphi_{1}^{*}$ is the estimated errors, $\sigma=\Delta_{p}+\widetilde{w}$.

Assumption 1. With $\sigma \in L_{2}[0, T], T \in(0,+\infty)$, there exists the constant $c_{0}$ such that $\sigma^{T} \sigma \leq c_{0}$.

Define a Lyapunov function

$$
V_{1}=\frac{1}{2} e_{p}^{T} e_{p}+\frac{1}{2}\left(\int_{0}^{t} e_{p} \mathrm{~d} t\right)^{T} \Lambda_{2}\left(\int_{0}^{t} e_{p} \mathrm{~d} t\right)+\frac{1}{2} \widetilde{\varphi}_{1}^{T} \widetilde{\varphi}_{1} .
$$
by

$$
\begin{aligned}
\dot{V}_{1}= & \frac{1}{2} \dot{e}_{p}^{T} e_{p}+\frac{1}{2} e_{p}^{T} \dot{e}_{p}+\frac{1}{2} e_{p}^{T} \Lambda_{2}\left(\int_{0}^{t} e_{p} \mathrm{~d} t\right)+\frac{1}{2}\left(\int_{0}^{t} e_{p} \mathrm{~d} t\right)^{T} \Lambda_{2} e_{p}+\dot{\tilde{\varphi}}_{1}^{T} \widetilde{\varphi}_{1} \\
\dot{V}_{1}= & \frac{1}{2}\left[-e_{p}^{T} A^{T} e_{p}-\left(\int_{0}^{t} e \mathrm{~d} t\right) \Lambda_{2}^{T} e_{p}+\widetilde{\varphi}_{1}^{T} \xi_{1}^{T} h^{T} B_{1}^{T} e_{p}+\sigma^{T} B_{1}^{T} e_{p}\right]+\frac{1}{2}\left[-e_{p}^{T} A^{T} e_{p}-e_{p}^{T} \Lambda_{2}\left(\int_{0}^{t} e_{p} \mathrm{~d} t\right)+e_{p}^{T} B_{1} h \xi_{1}^{T} \widetilde{\varphi}_{1}+e_{p}^{T} B_{1} \sigma\right] \\
& +\frac{1}{2} e_{p}^{T} \Lambda_{2}\left(\int_{0}^{t} e_{p} \mathrm{~d} t\right)+\frac{1}{2}\left(\int_{0}^{t} e_{p} \mathrm{~d} t\right)^{T} \Lambda_{2} e_{p}+\dot{\tilde{\varphi}}_{1}^{T} \widetilde{\varphi}_{1} . \\
\dot{V}_{1}= & -e_{p}^{T} A e_{p}+\frac{1}{2}\left(\sigma^{T} B_{1}^{T} e_{p}+e_{p}^{T} B_{1} \sigma\right)+\left(e_{p}^{T} B_{1} h \xi_{1}^{T}+\dot{\tilde{\varphi}}_{1}^{T}\right) \widetilde{\varphi}_{1} .
\end{aligned}
$$

Using (13), the following result is obtained:

$$
\dot{V}_{1}=-e_{p}^{T}(A-I) e_{p}-\left(e_{p}-\frac{1}{2} \sigma\right)^{T}\left(e_{p}-\frac{1}{2} \sigma\right)+\frac{1}{4} \sigma^{T} \sigma
$$

or

$$
\dot{V}_{1} \leq-e_{p}^{T} Q_{1} e_{p}+\frac{1}{4} \sigma^{T} \sigma .
$$

By integrating both sides of equation $(21)$ in $[0, T]$, the following equation is derived:

$$
V_{1}(T)-V_{1}(0) \leq-\int_{0}^{T} e_{p}^{T} Q_{1} e_{p} \mathrm{~d} t+\frac{1}{4} \int_{0}^{T} \sigma^{T} \sigma \mathrm{d} t
$$

According to Assumption 1, we have $\left\|\sigma^{2}\right\| \leq c_{0}$ and combining with the condition $V_{1}(T) \geq 0$, so $\int_{0}^{T} e_{p}^{T} Q_{1} e_{p} \mathrm{~d} t$ is bounded in $[0, T]$. As $V_{1}$ has all variables which are bounded, $V_{1}$ is bounded.
Let us consider function $V_{0}\left(e_{p}, t\right)$ as follows:

$$
V_{0}=e_{p}^{T} Q_{1} e_{p} \text {. }
$$

The time derivative of $V_{0}$ is given by

$$
\dot{V}_{0}=2 e_{p}^{T} Q_{1} \dot{e}_{p} .
$$

As $e_{p}, h, \xi_{1}$, and $\tilde{\varphi}_{1}$ are bounded and $\sigma$ is limited, $\dot{e}_{p}$ is bounded. According to Barbalat's lemma, $V_{0}\left(e_{p}, t\right) \longrightarrow 0$ as $t \longrightarrow \infty$, which means that error system (5) is asymptotically stable under control law (12).

2.2.2. Dynamic Controller Design. Considering the dynamic equation of WMR (4), multiplying both sides of (4) with $M^{-1}$, the following is obtained:

$$
\begin{aligned}
M^{-1} M \dot{v}= & \left(-M^{-1} B(v)\right) v+\left(-M^{-1}\right)\left(E v+Q \ddot{\gamma}+C \dot{\eta}+G \ddot{\eta}+\tau_{d}\right) \\
& +M^{-1} \tau,
\end{aligned}
$$


or

$$
\dot{v}=\left(-M^{-1} B(v)\right) v+M^{-1} \tau+D,
$$

where $D=\left(-M^{-1}\right)\left(E v+Q \ddot{\gamma}+C \dot{\eta}+G \ddot{\eta}+\tau_{d}\right)$.

Equation (26) can be shortened as

$$
\dot{v}=F(v)+G u_{m}+D,
$$

where $v=\left[\begin{array}{c}v_{R} \\ v_{L}\end{array}\right], \quad \dot{v}=\left[\begin{array}{c}\dot{v}_{R} \\ \dot{v}_{L}\end{array}\right], \quad F(v)=\left(-M^{-1} B(v)\right) v, G=$ $M^{-1}$, and the control input $u_{m}=\tau=\left[\begin{array}{ll}\tau_{R} & \tau_{L}\end{array}\right]^{T}=$ $\left[\begin{array}{ll}u_{m 1} & u_{m 2}\end{array}\right]^{T}$.

Define $\widetilde{e}=v-v_{\text {ref }}=v-u$. Considering the space-model equation (27) without disturbance $(D=0)$, the controller is proposed as follows:

$$
u_{m}^{*}=-G^{-1}\left(F(v)+\mathrm{K}_{2} \tilde{e}-\dot{u}\right),
$$

where $K_{2}$ is the positive scalar.

Substituting (28) into (27) leads to the following result:

$$
\dot{\tilde{e}}+K_{2} \widetilde{e}=0 \text {. }
$$

From (29), the control objective is achieved, that is, $\lim _{t \rightarrow \infty} \widetilde{e}(t)=0$.

Actually, in practice, disturbance $D$ is different from zero, so we cannot apply optimal control law (28). To deal with this problem, the following adaptive interval fuzzy typeII $H \infty$ controller is presented with the control law as follows:

$$
u_{m}=u\left(\widetilde{e} \mid \varphi_{2}\right)-G^{-1} u_{s}
$$

where $u\left(\widetilde{e} \mid \varphi_{2}\right)=\xi_{2}^{T}(\widetilde{e}) \varphi_{2}$ is the output of the adaptive interval fuzzy type-II and $\xi_{2}(\widetilde{e})=\operatorname{diag}\left(\left[\xi_{21}(\widetilde{e})\right.\right.$, $\left.\left.\xi_{22}(\widetilde{e}), \ldots, \xi_{2 N}(\widetilde{e})\right]\right), \xi_{2 i}=f_{2 i} / \sum_{i=1}^{N} f_{2 i}$ in which $f_{2 i}$ is the firing interval of the $i$-th rule, $N$ is the number of rules, $\varphi_{2}$ is the parameter updated by the adaptive law:

$$
\dot{\varphi}_{2}=-\left\{\gamma \xi_{2}^{T}(\widetilde{e}) G B_{2}^{T} P \widetilde{e}\right\}
$$

and $u_{s}=\left[\begin{array}{ll}u_{s 1} & u_{s 2}\end{array}\right]^{T}$ is Ho controller:

$$
u_{s}=\frac{1}{\beta} B_{2}^{T} P \widetilde{e},
$$

where $\beta$ and $\gamma$ are the positive constants and $P$ is the solution of the algebraic Riccati-like equation:

$$
P K_{2}+K_{2}^{T} P+Q_{2}-P B_{2}\left(\frac{2}{\beta}-\frac{1}{\varepsilon^{2}}\right) B_{2}^{T} P=0
$$

in which $\varepsilon$ is positive scalar and $Q_{2}$ is a prescribed symmetric-defined positive matrix.

From (28), the following result is obtained:

$$
F(v)=-G u_{m}^{*}-K_{2} \tilde{e}+\dot{u}
$$

Substituting (30) and (34) into (27), the result is as follows:

$$
\dot{\tilde{e}}=-K_{2} \widetilde{e}+B_{2} G\left(u\left(\widetilde{e} \mid \varphi_{2}\right)\right)-u_{m}^{*}+B_{2} D-B_{2} u_{s},
$$
where $B_{2}=\left[\begin{array}{ll}1 & 0 \\ 0 & 1\end{array}\right]$. The estimated error is determined as
follows:

$$
\Gamma=G\left(u\left(\widetilde{e} \mid \varphi_{2}^{*}\right)-u_{2}^{*}\right) .
$$

The closed-loop system error can be developed by substituting (36) into (35) as

$$
\dot{\tilde{e}}=-K_{2} \widetilde{e}+B_{2} G\left(u\left(\widetilde{e} \mid \varphi_{2}\right)-u\left(\widetilde{e} \mid \varphi_{2}^{*}\right)\right)+B_{2}(D+\Gamma)-B_{2} u_{s}
$$

or

$$
\dot{\tilde{e}}=-K_{2} \widetilde{e}+B_{2} G \xi_{2}^{T}(\widetilde{e}) \widetilde{\varphi}_{2}+B_{2}\left(\delta-u_{s}\right)
$$

where $\tilde{\varphi}_{2}=\varphi_{2}-\varphi_{2}^{*}$ and $\delta=D+\Gamma$.

\subsubsection{Overall Stability}

Assumption 2. In this work, we assume that the external disturbance $D$ is bounded, so there exists a constant $d_{0}$ such that $\varepsilon^{2} \delta^{T} \delta \leq d_{0}$.

Consider the Lyapunov function

$$
V_{2}=V_{1}+\frac{1}{2} \widetilde{e}^{T} P \widetilde{e}+\frac{1}{2 \gamma} \widetilde{\varphi}_{2}^{T} \widetilde{\varphi}_{2}
$$
by

The time of derivative of the Lyapunov function is given

$$
\dot{V}_{2}=\dot{V}_{1}+\frac{1}{2} \dot{\tilde{e}}^{T} P \widetilde{e}+\frac{1}{2} \widetilde{e}^{T} P \dot{\tilde{e}}+\frac{1}{\gamma} \dot{\tilde{\varphi}}_{2}^{T} \widetilde{\varphi}_{2} .
$$

Substituting (32) and (38) into (40), we have

$$
\begin{aligned}
\dot{V}_{2} & =\dot{V}_{1}+\frac{1}{2}\left[\widetilde{e}^{T} K_{2}^{T} P \widetilde{e}+\xi_{2}^{T}(\widetilde{e}) \widetilde{\varphi}_{2} G B_{2}^{T} P \widetilde{e}+\delta^{T} B_{2}^{T} P \widetilde{e}-\frac{1}{\beta} \widetilde{e}^{T} P B_{2} B_{2}^{T} P \widetilde{e}\right]+\frac{1}{2}\left[\widetilde{e}^{T} P K_{2} \widetilde{e}+\widetilde{e}^{T} P B_{2} G \widetilde{\varphi}_{2}^{T} \xi_{2}(\widetilde{e})+\widetilde{e}^{T} P B_{2} \delta-\frac{1}{\beta} \widetilde{e}^{T} P B_{2} B_{2}^{T} P \widetilde{e}\right]+\frac{1}{\gamma} \dot{\tilde{\varphi}}_{2}^{T} \widetilde{\varphi}_{2} \\
& =\dot{V}_{1}+\frac{1}{2} \widetilde{e}^{T}\left[P K_{2}+K_{2}^{T} P-\frac{2}{\beta} P B_{2} B_{2}^{T} P\right] \widetilde{e}+\frac{1}{2}\left(\delta^{T} B_{2}^{T} P \widetilde{e}+\widetilde{e}^{T} P B_{2} \delta\right)+\frac{1}{\gamma}\left(\dot{\tilde{\varphi}}_{2}^{T}+\gamma \widetilde{e}^{T} P B_{2} G \xi_{2}(\widetilde{e})\right) \widetilde{\varphi}_{2}
\end{aligned}
$$

Substituting the adaptive law (31) into (41), we get 


$$
\begin{aligned}
\dot{V}_{2} & =\dot{V}_{1}+\frac{1}{2} \widetilde{e}^{T}\left[-Q_{2}-\frac{1}{\varepsilon^{2}} P B_{2} B_{2}^{T} P\right] \widetilde{e}+\frac{1}{2}\left(\delta^{T} B_{2}^{T} P \widetilde{e}+\widetilde{e}^{T} P B_{2} \delta\right) \\
& =\dot{V}_{1}-\frac{1}{2} \widetilde{e}^{T} Q_{2} \widetilde{e}+\frac{1}{2} \varepsilon^{2} \delta^{T} \delta-\frac{1}{2}\left(\frac{1}{\varepsilon} \widetilde{e}^{T} P B_{2}-\varepsilon \delta\right)^{T}\left(\frac{1}{\varepsilon} \widetilde{e}^{T} P B_{2}-\varepsilon \delta\right) .
\end{aligned}
$$

Due to $1 / 2\left(1 / \varepsilon \widetilde{e}^{T} P B_{2}-\varepsilon \delta\right)^{T}\left(1 / \varepsilon \widetilde{e}^{T} P B_{2}-\varepsilon \delta\right) \geq 0$ and combining with the previous results of $V_{1}$, the following is obtained:

$$
\dot{V}_{2} \leq-e_{p}^{T} Q_{1} e_{p}+\frac{1}{4} \sigma^{T} \sigma-\frac{1}{2} \widetilde{e}^{T} Q_{2} \widetilde{e}+\frac{1}{2} \varepsilon^{2} \delta^{T} \delta .
$$

Integrating both of sides (43) in $[0, T]$, the following equation is derived:

$$
V_{2}(T)-V_{2}(0) \leq-\int_{0}^{T} e_{p}^{T} Q_{1} e_{p} \mathrm{~d} t-\frac{1}{2} \int_{0}^{T} \tilde{e}^{T} Q_{2} \widetilde{e} \mathrm{~d} t+\frac{1}{4} \int_{0}^{T} \sigma^{T} \sigma \mathrm{d} t+\frac{1}{2} \int_{0}^{T} \varepsilon^{2} \delta^{T} \delta \mathrm{d} t
$$

${ }^{T}$ From the condition $\varepsilon^{2}\left\|\delta^{2}\right\| \leq d_{0}, V_{2}(T) \geq 0$ and $\int_{0}^{T} e_{p}^{T} Q_{1} e_{p} \mathrm{~d} t>0$, the above inequality is equivalent to

$$
-V_{2}(0) \leq-\frac{1}{2} \int_{0}^{T} \widetilde{e}^{T} Q_{2} \widetilde{e} \mathrm{~d} t+\frac{1}{4} \int_{0}^{T} \sigma^{T} \sigma \mathrm{d} t+\frac{1}{2} \int_{0}^{T} \varepsilon^{2} \delta^{T} \delta \mathrm{d} t
$$

or

$$
\begin{aligned}
\frac{1}{2} \int_{0}^{T} \widetilde{e}^{T} Q_{2} \widetilde{e} \mathrm{~d} t \leq & \frac{1}{4} \int_{0}^{T} \sigma^{T} \sigma \mathrm{d} t+\frac{1}{2} \int_{0}^{T} \varepsilon^{2} \delta^{T} \delta \mathrm{d} t+V_{1}(0) \\
& +\frac{1}{2} \widetilde{e}^{T}(0) P \widetilde{e}(0)+\frac{1}{2 \gamma} \widetilde{\varphi}_{2}^{T}(0) \widetilde{\varphi}_{2}(0) .
\end{aligned}
$$

The proof is completed, and then the Hoo tracking performance can be achieved for a prescribed attenuation level $\varepsilon$.

\section{Control Strategy Verification}

The physical parameters of the WMR are given as $m_{G}=10 \mathrm{~kg}, \quad m_{w}=2 \mathrm{~kg}, \quad I_{w}=0.1 \mathrm{kgm}^{2}, \quad I_{D}=0.05 \mathrm{kgm}^{2}$, $I_{G}=4 \mathrm{kgm}^{2}, b=0.3 \mathrm{~m}, r=0.15 \mathrm{~m}$, and $a=0.2 \mathrm{~m}$.

Input disturbances and uncertainties as well as slipping and skidding parameters are assumed as

$$
\begin{aligned}
\tau_{d} & =[2+\sin (0.1 t) 1+\cos (0.1 t)]^{T}, \\
{\left[\dot{\gamma}_{R}, \dot{\gamma}_{L}, \dot{\eta}\right]^{T} } & = \begin{cases}{[0,0,0]^{T}(\mathrm{~m} / \mathrm{sec}),} & \text { for } t<5(\mathrm{sec}), \\
{[0.3 \sin (2 t), 0.3 \cos (2 t), 0.2]^{T}(\mathrm{~m} / \mathrm{sec}),} & \text { for } t \geq 5(\mathrm{sec}),\end{cases}
\end{aligned}
$$

The control gains are given as $\Lambda_{1}=2, \Lambda_{2}=3, p=0.25$, and $\gamma_{2}=0.0001 p^{2}$. The feedback gain matrix is selected as follows:

$$
\begin{aligned}
Q_{2} & =\left[\begin{array}{cc}
30 & 0 \\
0 & 30
\end{array}\right], \\
P & =\left[\begin{array}{cc}
6.5678 & 0 \\
0 & 6.5678
\end{array}\right], \\
K_{2}^{T} & =\left[\begin{array}{ll}
1 & 0 \\
0 & 1
\end{array}\right] .
\end{aligned}
$$

Next, the 4 rules of fuzzy type-II system are chosen for both inner and outer loops. The membership function is chosen as trapezoidal functions for $e_{p 1}, e_{p 2}$ and $\widetilde{e}_{1}, \widetilde{e}_{2}$. The fuzzy sets are constructed as follows:

$$
\begin{aligned}
& X_{1}=\left[\begin{array}{l}
X_{11 u} \\
X_{11 l} \\
X_{12 u} \\
X_{12 l}
\end{array}\right]=\left[\begin{array}{cccc}
-1,5 & -1,5 & -0,5 & 1,5 \\
-1,5 & -1,5 & -1,5 & 0,5 \\
-1,5 & -1,5 & 0,5 & 1,5 \\
-0,5 & -0,5 & 0,5 & 1,5
\end{array}\right], \\
& X_{2}=\left[\begin{array}{l}
X_{21 u} \\
X_{21 l} \\
X_{22 u} \\
X_{22 l}
\end{array}\right]=\left[\begin{array}{cccc}
-3 & -3 & -1 & 3 \\
-3 & -3 & 1 & 3 \\
-3 & -3 & 1 & 3 \\
-1 & -1 & 3 & 3
\end{array}\right], \\
& V_{1}=\left[\begin{array}{l}
V_{11 u} \\
V_{11 l} \\
V_{12 l} \\
V_{12 l}
\end{array}\right]=\left[\begin{array}{cccc}
-9 & -9 & -3 & 9 \\
-9 & -9 & -9 & 3 \\
-9 & 3 & 9 & 9 \\
-3 & 9 & 9 & 9
\end{array}\right], \\
& V_{2}=\left[\begin{array}{l}
V_{21 u} \\
V_{21 l} \\
V_{22 u} \\
V_{22 l}
\end{array}\right]=\left[\begin{array}{cccc}
-15 & -15 & -7.5 & 15 \\
-15 & -15 & -15 & 7.5 \\
-15 & 7.5 & 15 & 15 \\
-7.5 & 15 & 15 & 15
\end{array}\right] .
\end{aligned}
$$

where $X_{i j u}, X_{i j l}, V_{i j u}$, and $V_{i j l}$ are type-I fuzzy sets.

The simulation is done with two types of reference trajectory: elliptical and trifolium shapes. In each case, the responses of the proposed scheme are compared with the corresponding fuzzy type-I to evaluate the effectiveness of the proposed algorithm.

Case 1. The target $\mathrm{C}$ moves on an elliptical trajectory with the following equation:

$$
\left\{\begin{array}{l}
x_{C}=5 \cos \left(\frac{\pi}{10} t\right), \\
y_{C}=5 \sin \left(\frac{\pi}{10} t\right) .
\end{array}\right.
$$




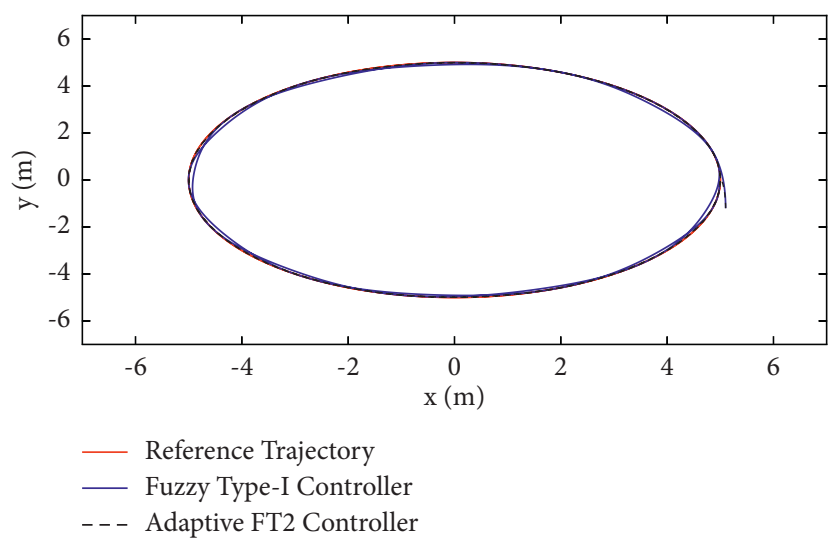

FIgure 3: The output trajectory of adaptive interval fuzzy type-II and T-S fuzzy type-I controller.

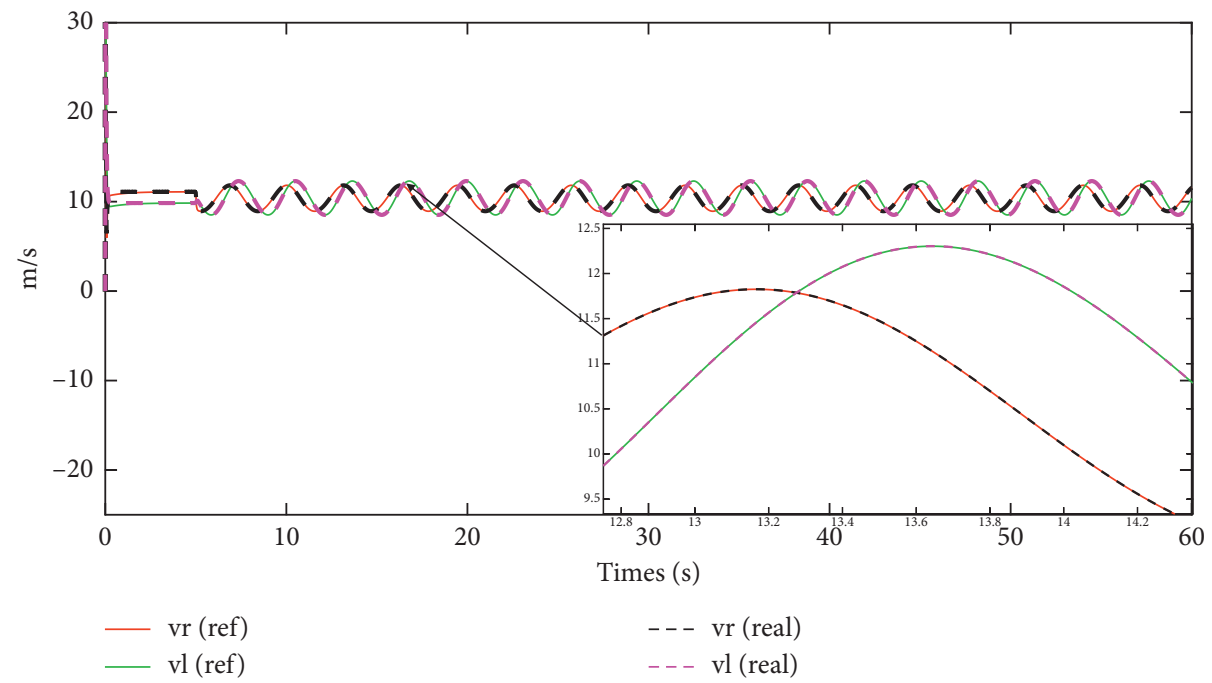

Figure 4: The real velocity of T-S fuzzy type-II controller for elliptical trajectory.

The initial values for system are chosen as $\left[x_{G}, y_{G}, \theta\right]=[5,-1, \pi / 2]$.

The results of the simulation are presented in Figures 3-5. Specifically, Figure 3 describes the reference trajectory, the response of the adaptive interval fuzzy typeII controller, and the response of the fuzzy type-I controller. It is easy to see in Figure 3 that, the trajectory of the WMR which is controlled by the proposed controller is more exact than the fuzzy type-I, i.e., the max error tracking of the proposed controller is $0.03 \mathrm{~m}$ and the fuzzy type-I controller is $0.0806 \mathrm{~m}$. Figures 4 and 5 are the velocity errors of the adaptive fuzzy type-II and fuzzy type-I controllers, respectively. The error between the real velocity and the reference velocity of the proposed scheme is nearly zero (Figure 4), while this error in the fuzzy type-I controller is so large (Figure 5), i.e., in Figure 5, the real velocity oscillates around the reference velocity with an amplitude of $0.02 \mathrm{~m} / \mathrm{s}$.
Case 2. The reference trajectory is trifolium which is formulated as follows:

$$
\left\{\begin{array}{l}
x_{C}=5 \cos \left(\frac{\pi}{10} t\right) \cos \left(\frac{\pi}{30} t\right), \\
y_{C}=5 \sin \left(\frac{\pi}{10} t\right) \sin \left(\frac{\pi}{30} t\right) .
\end{array}\right.
$$

The initial values for system are chosen as $\left[x_{G}, y_{G}, \theta\right]=[5,-1, \pi / 2]$.

The simulation results are shown in Figures 6-8. Similar to Case 1, we also see that responses of the proposed controller is better, i.e., the maximum error tracking of the proposed controller is $0.03 \mathrm{~m}$ and this one is $0.0807 \mathrm{~m}$ for fuzzy type-I controller. In Figure 7, the difference between the real velocity and the reference velocity is almost zero, while the real velocity of the fuzzy type-I controller is affected by external disturbance, and the oscillation is so large 


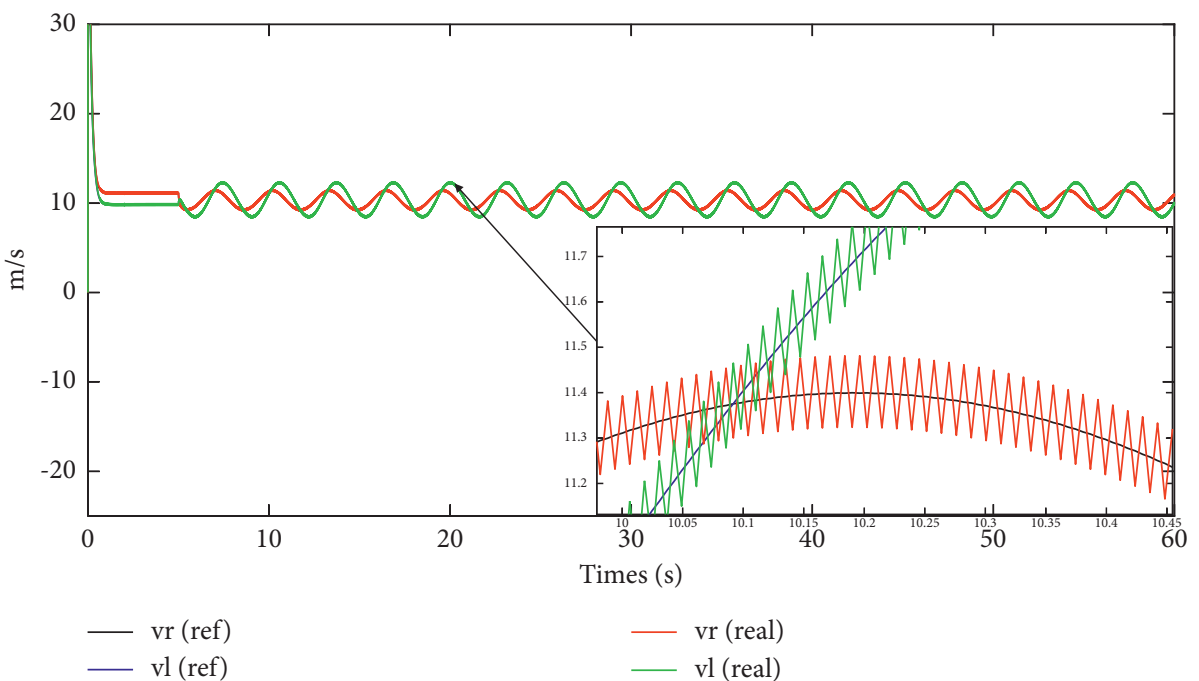

Figure 5: The real velocity of adaptive T-S fuzzy type-I for elliptical trajectory.

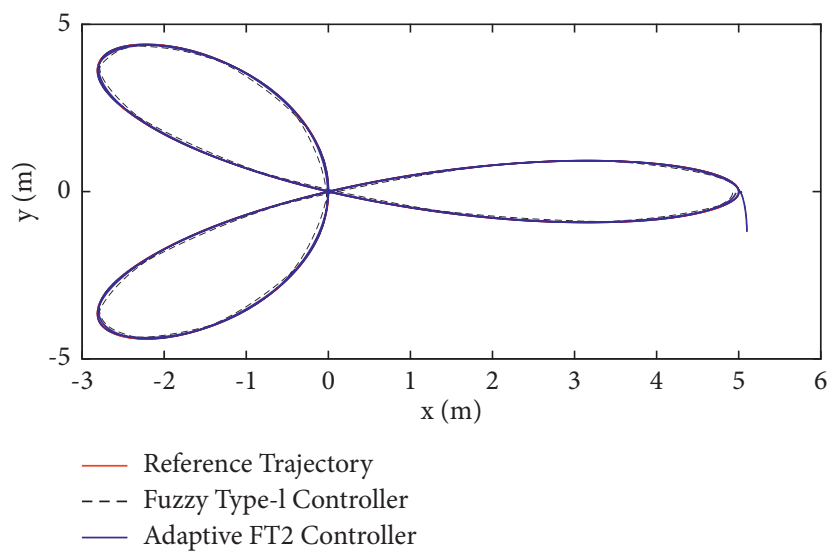

FiguRE 6: The output trajectory of adaptive interval fuzzy type-II and T-S fuzzy type-I controller for trifolium trajectory.

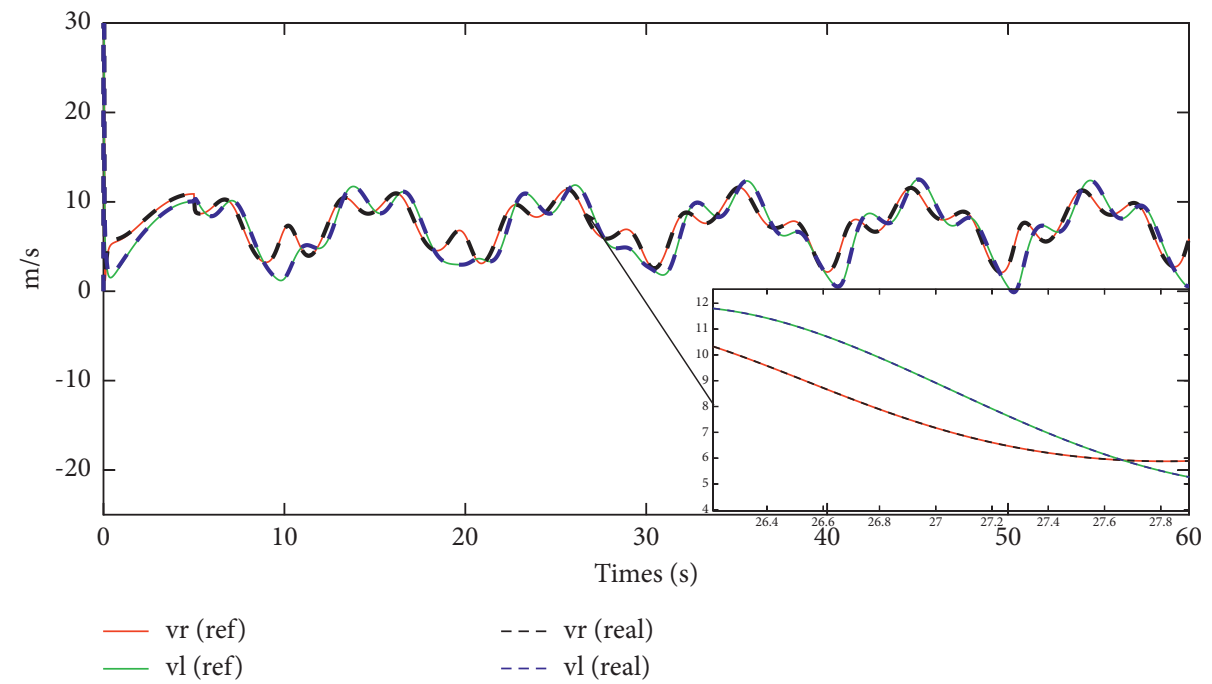

FiguRE 7: The real velocity of adaptive interval fuzzy type-II for trifolium trajectory. 


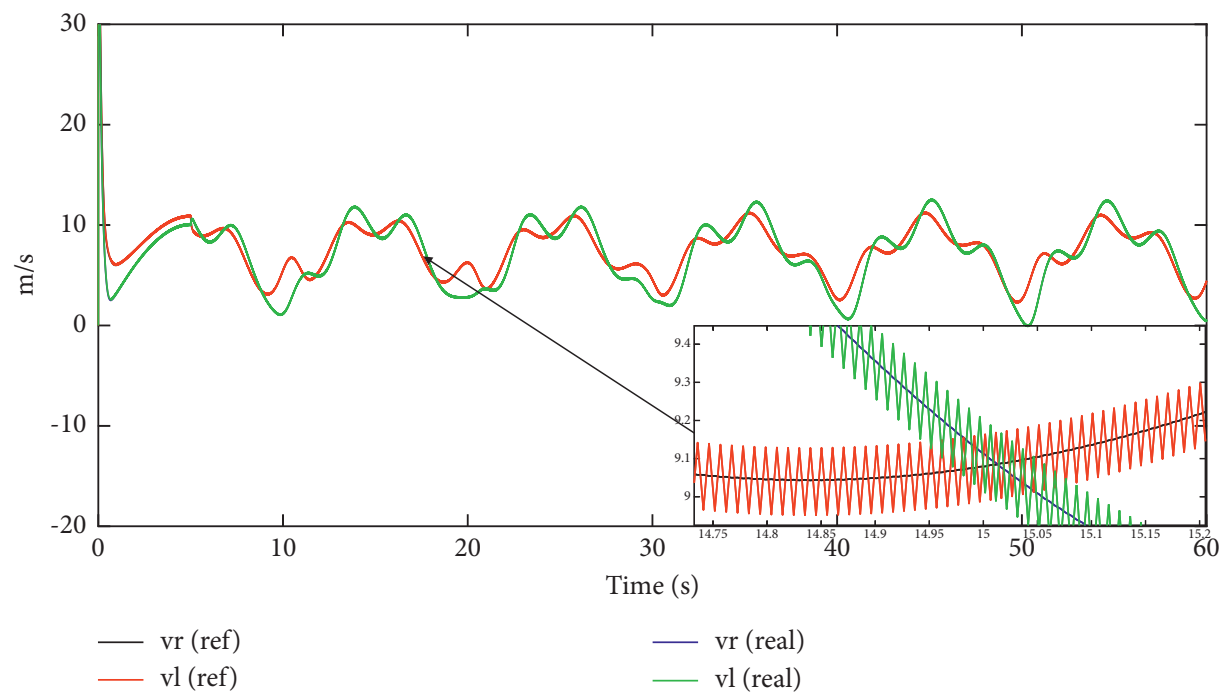

FIGURE 8: The real velocity of T-S fuzzy type-I for trifolium trajectory.

TABLE 1: Numerical comparison between the proposed controller and T-S fuzzy type-I controller.

\begin{tabular}{lcccc}
\hline & \multicolumn{2}{c}{ Trifolium } & \multicolumn{2}{c}{ Elliptical } \\
& Tracking error $(\mathrm{m})$ & Velocity $(\mathrm{m} / \mathrm{s})$ & Tracking error $(\mathrm{m})$ & Velocity $(\mathrm{m} / \mathrm{s})$ \\
\hline Proposed controller & $\operatorname{Max}=0.03$ & No oscillation & Max $=0.03$ & No oscillation \\
Fuzzy type-I controller & $\operatorname{Max}=0.0806$ & Oscillation & Max $=0.0807$ & Oscillation \\
\hline
\end{tabular}

with an amplitude about $0.1 \mathrm{~m} / \mathrm{s}$ (Figure 8). From these simulation results, we can see that the adaptive interval fuzzy type-II logic controller can handle unknown wheel slipping and skidding, uncertainties, and unknown bounded external disturbance better than the fuzzy type-I logic controller.

All of the above analyses are concluded in Table 1.

\section{Conclusions}

In this paper, an adaptive fuzzy type-II controller was proposed for the WMR system under the condition of wheel slips and external disturbances. The control system included the dynamic loop and kinematic loop. For each loop, this proposed controller was used to compensate for the unknown uncertainties and disturbances. In this work, the stability of the closed-loop system and the convergence of the trajectory-tracking errors were mathematically proved based on the Lyapunov theory. The properness of the proposed controller was verified through two types of reference trajectory. Also, the responses of the proposed controller were compared with the fuzzy type-I controller's one to prove the effectiveness of the illustrated scheme. The simulation results showed that the performance of the adaptive fuzzy type-II controller was better than the fuzzy type-I controller, i.e., smaller error, smoother velocity, and smaller oscillation even that the trajectory was complicated. However, the disadvantage of the proposed scheme is the complication in the mathematic proof. This complication is normal for two-loop (cascade) control structure. Moreover, the control model in each loop is imperfect, and then the fuzzy controller component with an adaptive parameter is added into each loop to deal with the uncertainties and disturbances. In the future, we have planned to develop the one loop control structure to reduce the complexity for the overall system.

\section{Data Availability}

The data used to support the findings of this study have been deposited in the CRC Press repository (https://www.routledge. com/Mobile-Robots-Inspiration-to-Implementation-SecondEdition/Jones-Seiger-Flynn/p/book/9780367447656); Robotics and Autonomous Systems repository (https://doi.org/10.1016/ j.robot.2016.01.002); International Conference on Control, Automation and Systems repository (DOI: 10.1109/ ICCAS.2015.7365010); IEEE Transactions on Industrial Electronics repository (DOI: 10.1109/TIE.2013.2282594); the Mediterranean Conference on Control and Automation repository (DOI: 10.1109/MED.2009.5164519); International Journal of Advanced Robotic Systems repository (https://doi. org/10.5772/55059); IEEE/RSJ International Conference on Intelligent Robots and Systems repository (DOI: 10.1109/ IROS.2010.5651060); IEEE Transactions on Systems, Man, and Cybernetics: Systems repository (DOI: 10.1109/ TSMC.2017.2677472); IEEE Transactions on Control Systems Technology repository (DOI: 10.1109/TCST.2008.922584); IET Control Theory Amp Applications repository (DOI: 10.1049/ iet-cta.2010.0026); IEEE Access repository (DOI: 10.1109/ ACCESS.2018.2862163); IEEE International Conference on Control and Robotics Engineering repository (DOI: 10.1109/ ICCRE.2016.7476148); Neurocomputing repository (DOI: https://doi.org/10.1016/j.neucom.2017.12.051); Nonlinear 
Dynamics repository (DOI: https://doi.org/10.1007/s11071019-05077-4); IEEE Transactions on Neural Networks and Learning Systems repository (DOI: 10.1109/ TNNLS.2019.2912082); Control Engineering Practice repository (https://doi.org/10.1016/S0967-0661(01)00126-5); IEEE Transactions on Fuzzy Systems repository (DOI: 10.1109/ TFUZZ.2019.2959972). IEEE International Conference on Granular Computing repository (DOI: 10.1109/GrC.2010.112); Journal of Central South University of Technology repository (https://doi.org/10.1007/s11771-011-0760-0); and International Journal of Control repository (https://doi.org/10.1080/ 00207179.2018.1458156).

\section{Conflicts of Interest}

The authors declare that there are no conflicts of interest regarding the publication of this paper.

\section{References}

[1] J. L. Jones, B. A. Seiger, and A. M. Flynn, Mobile Robots: Inspiration to Implementation, CRC Press, Baco Raton, FL, USA, 2019.

[2] L. Xin, Q. Wang, J. She, and Y. Li, "Robust adaptive tracking control of wheeled mobile robot," Robotics and Autonomous Systems, vol. 78, pp. 36-48, 2016.

[3] S. Tchenderli-Baham, F. Hamerlain, and N. Saadia, "Adaptive sliding mode for the control of a wheeled mobile robot," International Conference on Control, Automation and Systems, pp. 699-703, 2015.

[4] J.-X. Xu, Z.-Q. Guo, and T. H. Lee, "Design and implementation of integral sliding-mode control on an underactuated two-wheeled mobile robot," IEEE Transactions on Industrial Electronics, vol. 61, pp. 3671-3681, 2013.

[5] D. Nganga-Kouya and F. A. Okou, "Adaptive backstepping control of a wheeled mobile robot," in Proceedings of the Mediterranean Conference On Control And Automation, pp. 85-91, Athens, Greece, July 2009.

[6] E.-J. Hwang, H.-S. Kang, C.-H. Hyun, and M. Park, "Robust backstepping control based on a lyapunov redesign for skidsteered wheeled mobile robots," International Journal of Advanced Robotic Systems, vol. 10, 2013.

[7] Y. Liang, L. Xu, R. Wei, and H. Hu, "Adaptive fuzzy control for trajectory tracking of mobile robot," in Proceedings of the IEEE/RSJ International Conference on Intelligent Robots and Systems, pp. 4755-4760, Prague, Czech Republic, October 2010.

[8] L. Ding, S. Li, Y.-J. Liu, H. Gao, C. Chen, and Z. Deng, "Adaptive neural network-based tracking control for full-state constrained wheeled mobile robotic system," IEEE Transactions on Systems, Man, and Cybernetics: Systems, vol. 47, no. 8, pp. 2410-2419, 2017.

[9] B. S. Park, S. J. Yoo, J. B. Park, and Y. H. Choi, “Adaptive neural sliding mode control of nonholonomic wheeled mobile robots with model uncertainty," IEEE Transactions on Control Systems Technology, vol. 17, pp. 207-214, 2008.

[10] S. J. Yoo, "Adaptive tracking control for a class of wheeled mobile robots with unknown skidding and slipping," IET Control Theory \& Applications, vol. 4, no. 10, pp. 2109-2119, 2010.

[11] S. Peng and W. Shi, "Adaptive fuzzy output feedback control of a nonholonomic wheeled mobile robot," IEEE Access, vol. 6, pp. 43414-43424, 2018.
[12] S.-H. Yu, C.-H. Hyun, and H. S. Kang, "Robust dynamic surface tracking control for uncertain wheeled mobile robot with skidding and slipping," IEEE International Conference on Control and Robotics Engineering, pp. 1-4, 2016.

[13] S. Li, L. Ding, H. Gao, C. Chen, Z. Liu, and Z. Deng, "Adaptive neural network tracking control-based reinforcement learning for wheeled mobile robots with skidding and slipping," Neurocomputing, vol. 283, pp. 20-30, 2018.

[14] M. Bucolo, A. Buscarino, C. Famoso, L. Fortuna, and M. Frasca, "Control of imperfect dynamical systems," Control of Imperfect Dynamical Systems Nonlinear Dynamics, vol. 98, no. 4, pp. 2989-2999, 2019.

[15] H. Wang, P. X. Liu, J. Bao, X. Xie, and S. Li, "Adaptive neural output-feedback decentralized control for large-scale nonlinear systems with stochastic disturbances," IEEE Transactions on Neural Networks and Learning Systems, pp. 972-983, 2019.

[16] M. Bucolo, L. Fortuna, M. Nelke, A. Rizzo, and T. Sciacca, "Prediction models for the corrosion phenomena in Pulp \& Paper plant," Control Engineering Practice, vol. 10, no. 2, pp. 227-237, 2002.

[17] M. Chen, H. Wang, and X. Liu, "Adaptive fuzzy practical fixed-time tracking control of nonlinear systems," IEEE Transactions on Fuzzy Systems, pp. 664-673, 2019.

[18] D. Wu and W. W. Tan, "Interval type-2 fuzzy pi controllers: why they are more robust," IEEE International Conference on Granular Computing, pp. 802-807, 2010.

[19] H.-B. Zhou, H. Ying, and J.-A. Duan, "Adaptive control using interval type-2 fuzzy logic for uncertain nonlinear systems," Journal of Central South University of Technology, vol. 18, 2011.

[20] T. Nguyena, T. Hoang, M. Pham, and N. Dao, "A Gaussian wavelet network-based robust adaptive tracking controller for a wheeled mobile robot with unknown wheel slips," International Journal of Control, vol. 92, no. 11, pp. 2681-2692, 2019. 